\title{
Paradis colonial
}

Bien que la présence européenne aux Amériques date déjà de plus d'un siècle lorsque Du Tertre se rend aux Antilles, jusqu'à l'intervention de Colbert en 1664, l'établissement des Français aux Îles reste marqué par ce désordre, rappelant ce chaos que Serge Gruzinski décrit dans les premières étapes de la conquête de l'Amérique par les Espagnols : on cherche à dominer un territoire sur les ruines de l'ordre qui y régnait antérieurement ${ }^{1}$. La disparition de cet ordre crée des zones floues où ce qui est autochtone se mélange avec ce que les Européens apportent de l'Ancien Monde. Comme pour contrebalancer la démesure qui marque le début de la colonisation, la description géographique des Antilles va chercher son point de départ bien loin de l'espace archipélagique des Amériques. Ainsi commence l'Histoire générale des Isles, dans l'édition Langlois de 1654:

I'ay souvent admiré dans l'Antiquité prophane l'avanture de deux petits jumeaux nouvellement nez, qui apres avoir esté jettez dans le Tybre, recueillis par une louve, qui leur fit office de mere, \& élevez dans une cabane de Berger; ont esté comme la semence feconde qui a produit ce grand arbre de l'Empire Romain, dont les branches se sont étenduës \& multipliées par l'Univers. (1654, tome I : 1-2)

En bon lecteur de Pierre le Martyr2, Du Tertre prend du recul pour présenter les Îles à travers le filtre d'un imaginaire antique. Ce cadre familier confère du sens à l'étrangeté. Partir de la fondation de Rome n'est pas anodin : l'empire français aspire alors à prendre la place de l'empire romain comme berceau de l'Église et de la civilisation occidentale. La deuxième édition de l'Histoire générale des Antilles, celle publiée par Jolly en 1667 , suggère que la stratégie a réussi. Il n'est plus alors question d'inscrire la colonie française dans une mythologie antique et biblique. Au contraire, l'histoire de l'établissement prend directement sa source dans la politique coloniale contemporaine. Ce changement annonce

1 Serge Gruzinski, La Pensée métisse, Paris, Fayard, 1999, p. 68 ; voir aussi p. 54-56.

2 Voir Antonello Gerbi, Nature in the New World: From Christopher Columbus to Gonzalo Fernández de Oviedo, Pittsburgh, University of Pittsburgh Press, 1985, p. 62-63. Pierre le Martyr introduit deux tropes importants: la mise en question de la critique de la nature américaine et la vision du Nouveau Monde comme continuation de l'Antiquité, p. 71. 
une attitude fondamentalement différente par rapport au projet colonial. Pendant les 13 ans qui séparent les 2 éditions, la France a consolidé sa présence en Amérique et elle a changé de règne depuis la majorité de Louis XIV et la politique de Colbert. En 1667, c'est effectivement le fait colonial qui forme la base de la relation, affirmant par là aussi le succès de cette entreprise.

Le lien avec l'Antiquité a aussi une portée épistémologique. Il serait à comprendre comme une tentative pour inscrire les Îles dans l'histoire et la mythologie européennes, afin de rompre avec l'imaginaire de la Renaissance qui faisait des Amériques un espace étrange et mystérieux, un vide à remplir. «Le chroniqueur arrache l'histoire des Antilles à son enracinement flibustier pour la situer dans une perspective humaniste et providentielle », écrit Ouellet ${ }^{3}$. Frank Lestringant parle lui d'un mouvement circulaire, rattachant l'Ancien Monde au Nouveau : à travers les renvois à l'Antiquité, le voyageur rend compte du gouffre de l'Amérique pour l'intégrer dans une symbolique portée par le roi ${ }^{4}$. L'espace américain démythifié permet une description plus objective, moins fantaisiste, de la nature qui lui est propre. Un discours conquérant accompagne l'opération, marqué par l'emploi de symboles destinés à donner l'impression que le règne français est prédestiné. Signe d'une transition conceptuelle dont les Îles font l'objet, le rattachement à une histoire mythologique européenne suggère que la colonisation elle-même subit ce que Lestringant appelle ailleurs un «processus d'allégorisation». La lutte des premiers habitants acquiert un plus haut sens, qui la rattache à l'histoire de la nation. Ce genre de passage «fait du Nouveau Monde, non pas exactement le miroir fidèle, mais l'idéal perdu de l'Ancien ${ }^{5}$.»

Il s'agit ici d'abord d'une sorte d'hétérochronie selon laquelle, d'après l'expression de Thomas Pavel, le missionnaire perçoit « le temps présent comme organiquement rattaché au temps de jadis 6 .» Un tel dédoublement n'est pas rare. Remarquons pourtant que l'idéal perdu de l'Ancien Monde ne fait pas chez Du Tertre l'objet d'une déploration. Au contraire, il sert à construire un pont entre les deux mondes, entre une mythologie antique et une nouvelle

3 Réal Ouellet, La Relation de voyage en Amérique (XVI ${ }^{e}-X V I I I^{e}$ siècles) : Au carrefour des genres, Québec, Presses de l'Université Laval/éditions du CIERL, 2010, p. 45. Marie-Christine Pioffet propose que le renvoi à l'Antiquité « implique toujours quelque regret du monde initial, un désir de ressourcement », La Tentation de l'épopée, p. 38.

4 Frank Lestringant, « Fictions de l'espace brésilien à la Renaissance :l'exemple de Guanabara », Arts et légendes d'espace : Figures du voyage et rhétoriques du monde, Christian Jacob et Frank Lestringant (dir.), Paris, Presses de l'école normale supérieure, 1981, p. 232.

5 Id., « L'Exotisme en France à la Renaissance », p. 12.

6 Thomas Pavel, L'Art de l'éloignement: Essai sur l'imagination classique, Paris, Gallimard, 1996, p. 37 . 
mythologie coloniale à composer. Dans l'Histoire générale des Antilles, cela s'exprime dans des passages comparatifs où des épisodes tirés de la mythologie grecque et biblique sont convoqués pour mettre en relief une sorte de contradiction merveilleuse entre la difficulté de la vie aux Îles et la production des richesses dans la colonie. Les habitants auraient par exemple subi le même sort que les Israélites pendant l'Exode; ailleurs, la colonie flotte sur l'océan caribéen comme Moïse sur le Nil. (1654, tome I : 17) À l'instar de toutes les figures mythiques choisies par Du Tertre parce qu'elles triomphent de leurs difficultés, la petite colonie, qui ressemble au départ à un bout de terre perdu dans l'océan entre trois continents, est appelée à dominer le Nouveau Monde ${ }^{7}$. On dirait en effet que, suivant le modèle de la renovatio de la Renaissance, le missionnaire cherche non pas seulement à reproduire l'idéal antique perdu, mais à le réinventer dans le cadre du Nouveau Monde. Ce dédoublement du temps est essentiel à la figuration de l'étranger. En effet, comme l'a bien montré François Moureau, l'exotisme «n'est pas spatial, il se fonde sur un rapport au temps qui annule celui que l'homme classique aurait pu entretenir avec l'espace - major e longinquo reverentia. C'est l'Antiquité qui est le point de fuite, la mise en perspective de l'ici et de l'ailleurs, plus que d'éventuels lieux ou mœurs du temps présent ${ }^{8}$ ». L'Antiquité et la Bible (notamment l'Ancien Testament) interviennent comme un truchement entre deux ordres spatiaux incompatibles, et c'est à partir de cette mise en perspective que le relateur peut écrire un lieu où cohabitent le même et l'étranger.

Construite sur les réminiscences de l'Antiquité perdue, cette nouvelle communauté est partagée entre la France et les Antilles. De là résulte un nouveau mythe, propre à une colonisation française dont la promotion se fonde sur l'imaginaire paradisiaque. Les notions associées à l'existence «sauvage » - la liberté, l'égalité - sont ensuite utilisées pour décrire la vie des habitants français des Îles. Ici, nous promet le missionnaire, «point de difference de Noble, \& de Routier, entre les habitans, celuy qui a plus de bien est plus considéré ; car il n'y a que les Officiers qui tiennent rang, ainsi les richesses seules font la distinction entre les autres ». (1667, tome II :474) Plus loin dans le même passage, Du Tertre précise que « [1] a façon de vivre du pays est si agreable, \& l'on y vit dans une liberté si honneste, que ie n'ay pas veu un seul homme, ny une seule femme qui en soient revenus, en qui ie n'aye remarqué une grande passion d'y retourner ». $(1667$, tome II : 476) Le même topos se laisse lire dans un proverbe plaisant, qui affirmerait que

7 Lallusion fait aussi référence au roi-enfant, Louis XIV. Nous remercions Frédéric Tinguely de nous l'avoir signalé.

8 François Moureau, Le Théâtre des voyages, p. 57. 
[...] les Isles estoient l'enfer des hommes François, \& le Paradis de leurs femmes; \& au contraire, qu'elles estoient l'enfer des femmes Sauvages, \& le Paradis de leurs maris; parce que les femmes Sauvages travaillent comme des esclaves, \& font tout, pendant que leurs maris se promenent ou s'arrachent la barbe ; \& que les François s'éventrent à force de travailler, pendant que leurs femmes ne se mettent en peine que de faire les belles. (1667, tome II : 475)

Le comique repose sur le filage de la métaphore paradisiaque, mais il se présente aussi comme un trope interculturel qui permet de rapprocher les différents modes de vie des habitants des Îles. On trouve en effet dans le portrait de la nouvelle communauté coloniale plusieurs passages où le mode de vie autochtone vient se superposer à la description de la société coloniale française, remodelée selon le modèle républicain hérité de Platon, et en fonction de l'image de l'âge d'or empruntée à Ovide. Ce que la société coloniale emprunte aux Autochtones, c'est surtout leur liberté, notion articulée en opposition au système sociétal européen et en lien étroit avec la notion de propriété. Aux Îles, la hiérarchie sociale n'est ni arbitraire ni donnée de droit; elle est un produit de la nature : on améliore son sort et on se crée une position d'éminence grâce aux richesses accumulées par le travail et non pas par héritage. Une telle liberté sauvage se lit comme le résultat de la vie dans un espace naturel tropical et insulaire qui n'est pas territorialisé ni objet de propriété comme il l'est en Europe. Par conséquent, les Français transplantés de l'Ancien Monde aux Îles peuvent aussi espérer jouir « d'une plus grande liberté que ceux qui restaient chez eux ${ }^{9}$.» En s'adaptant à l'environnement, la société coloniale retrouverait le naturel que l'Europe avait perdu.

\section{Construire un paradis}

La vision de la nouvelle communauté coloniale sous ses traits paradisiaques apparait, elle aussi, dans les parties introductives, pour faire écho à la représentation édénique du «bon sauvage». Une image utopique de la vie aux Îles précède aussi la description des habitants français, comme si l'idéalisation du «sauvage » (naturel et humain) venait se greffer à la représentation de la colonisation. Il s'agit d'une fiction destinée à former ce que l'on pourrait appeler, pour reprendre l'expression du sociologue Benedict Anderson, une

9 Geoffrey Atkinson, Les Relations de voyage au XVII esiècle, p. 35 . 
« communauté imaginaire », fondant un discours nationaliste avant la lettre ${ }^{10}$. Dans le cadre d'une telle reconstruction identitaire et géographique, et à travers les réitérations d'un discours sur la nature sauvage, Du Tertre élabore sa propre variante du discours paradisiaque.

Dans l'introduction à la première partie de la première édition de l'Histoire générale des Antilles, Du Tertre plonge en effet son lecteur dans une géographie symbolique ancrée dans l'imaginaire caribéen :

Mais ie puis dire, sans rien donner à la flatterie, que l'establissement de nostre Colonie Françoise dans les Isles Cannibales n'est pas moins émerveillable, ny moins étonnant. Car si nous considerons avec attention son commancement \& son progrez, nous la verrons naistre comme une petite source, qui se dégorgeant insensiblement par des voyes connuës seulement de Dieu, malgré les obstacles des montagnes, \& les contradictions des hommes, va innonder les plus belles terres de l'Amerique. Elle vous semblera d'abord rünée tout à fait dans sa naissance, \& vous remarquerez en mesme temps, que recueillant les pieces de son débris, elle se restablit sur ses propres ruynes contre toute sorte d'esperance, \& avec tant d'avantage \& tant de succez, que toute abandonnée \& toute persecutée, mesme qu'elle estoit de ceux qui la devoient maintenir, elle remplit desia d'habitans François plusieurs belles terres capables de composer autant de Provinces. (1654, tome I : 2 )

On peut ici détecter un mouvement de retour à la géographie antillaise après le détour par l'Antiquité sur lequel s'ouvre la relation. Cette nouvelle mythologie propre à la région se distingue du primitivisme qui caractérise l'imaginaire paradisiaque lié au sauvage. Le «petit paradis toujours verdoyant» est maintenant associé à la férocité cannibale. Il ne s'agit plus de saisir le décor d'une nature tropicale édénique, mais d'admirer la transformation des Îles elles-mêmes, de l'état de nature sauvage à l'état de nature cultivée.

À la différence de l'image paradisiaque brossée au début de la description des Autochtones, cette version du paradis se déroule dans une temporalité étroitement liée à la colonisation, loin de la stagnation temporelle de la nature édénique sauvage ${ }^{11}$. Les marqueurs temporels du futur préparent le lecteur à

10 Nous nous référons ici à l'ouvrage de Benedict Anderson Imagined Communities: Reflections on the Origin and Spread of Nationalism, édition révisée, New York, Verso, 2016 [1983].

11 Pour une analyse des dimensions temporelles dans l'ouvrage de Du Tertre voir Christina Kullberg, "Like Moses on the Nile": Competing Temporalities in Jean-Baptiste Du Tertre's Histoire générale des Antilles habitées par les François (1654/1667)», Time and 
ce qui va venir en même temps qu'ils orientent la description vers le progrès. «Létablissement de nostre Colonie Françoise» est comparé à une source, un élément naturel associé à l'imaginaire paradisiaque et fondamental de la fondation d'une communauté. L'analogie permet d'inscrire la colonie dans le paysage antillais, mais le site qu'évoque Du Tertre rappelle autant le paysage antique d'une toile bucolique de la Renaissance que l'espace naturel des îles tropicales. Les éléments constitutifs de ce paysage sont des ruines architecturales, dont les morceaux et les débris suggèrent l'existence d'un passé glorieux, comme des vestiges antiques dans un paysage latin. La force du rapprochement ne vient pas tant de ce qu'il permet de donner vie aux habitants, mais de ce qu'il permet d'accumuler pour les réemployer les « pieces de son débris »tout comme la France accumule et crée à partir des ruines laissées par les Espagnols, et améliore ainsi les Îles jusqu'à la perfection. L'opération repose sur une double temporalité : les ruines évoquent le passé avec une pointe de nostalgie, tandis que le discours lui-même s'oriente vers l'avenir. Curieusement, c'est dans l'élan vers l'avenir, la naissance (évoquée deux fois) et le lent progrès de la colonie que s'ouvre un autre espace paradisiaque, celui des «plus belles terres capables de composer autant de Provinces ».

Cette vision est aussi codifiée que l'idéalisation du bon sauvage et rompt d'avec la narration factuelle. Mais si ces parties introduisant les descriptions des Amérindiens s'inscrivent dans une tradition livresque et viatique, l'enjeu des passages offrant la vision idéalisée de la communauté coloniale est différent. Si le relateur utilise des références analogues, c'est cette fois pour fonder un nouveau discours. On le voit clairement dans la longue introduction qu'il donne à la partie consacrée à la description des habitants (français) des Îles dans l'édition de 1667 :

L'on peut facilement juger, parce que j'ay dit dans la premiere Partie de cette Histoire, en faisant le recit de l'establissement des Colonies Françoises, qu'à les considerer dans cét estat, \& dans celuy auquel elles sont aujourd'huy, il y a quelque rapport à ce qui arriva au commencement du monde: qui n'estant qu'une masse confuse, \& sans agrément, n'eut pas plustost receu l'ordre \& la disposition que la divine Sagesse y establit, qu'il parut un ouvrage digne de la puissance qui l'avoit tiré du neant. En effet, c'est à peu prez de cette maniere que l'on doit parler de nos Colonies, tirées ce semble du neant, à cause de la foiblesse de leurs commencemens, qui nous ont fait voir tant de confusions \& de desordres,

Temporalities in European Travel Writing, Paula Henrikson et Christina Kullberg (dir.), London, Routledge, à paraitre. 
qu'elles ressembloient veritablement à un cahos remply de tenebres, qui n'a esté démeslé dans la suite des temps, qu'avec des peines incroyables \& des travaux infinis : \& si nous les voyons maintenant dans un estat pareil à celuy du monde, lors qu'il fut éclairé de la lumiere du Soleil, nous en avons l'obligation à nostre triomphant Monarque, qui à guise d'un Soleil éclatant porte ses rayons dans ces Pays éloignez, par les soins extraordinaires qu'il prend de les conserver, \& de les maintenir dans la paix \& dans le repos, \& d'y faire naistre l'abondance de toutes sorte de biens. (1667, tome II : 419-420)

L'analogie entre la fondation de Rome et l'établissement des colonies françaises paraît à présent bien modeste à côté de cette comparaison avec la création du monde. C'est en quelque sorte le «geste de découverte » (gesture of discovery) dont parlait Hulme ${ }^{12}$, poussé à l'extrême : si les Espagnols découvrent de nouvelles terres, les Français créent un monde lorsqu'ils mettent en ordre la masse confuse de ces terres nouvelles. Dans cette optique, Du Tertre introduit dans sa relation une temporalité supplémentaire, qui ne serait ni celle de l'Antiquité, ni celle des îles précoloniales placées hors du temps, mais une temporalité qui relie l'historicité européenne au vide achronique dans lequel existeraient les peuples amérindiens : le temps futur de la colonisation. C'est ce temps, celui du progrès introduit par les colons (français) capables de démêler tel «cahos remply de tenebres », qui ordonnera toute l'écriture et permettra la reconfiguration de l'exotisme.

Cette forme d'historicité qui l'emporte sur le spatial afin d'introduire dans l'ordre de l'écriture la productivité et le progrès réapparaît aussi dans les passages qui introduisent les descriptions topographiques des Îles. C'est comme si Du Tertre cherchait à diminuer la portée de l'espace, qui tend à dominer le discours descriptif de la topographie, en encadrant son évocation par le récit du développement de l'île :

Il est vray que dans ce premier estat, ces Pays n'avoient rien que de rebutant. Les peuples qui les habitoient estoient Barbares, les Terres incultes, ne produisoient rien qu'apres un travail inconcevable, \& les vaisseaux n'ayant point accoustumé de les frequenter, nos premiers François perissoient souvent, par la main de ces Barbares, succomboient sous le faix du travail, ou manquoient des choses qui leur devoient estre apportées de dehors. Mais depuis que les Sauvages ont esté rangés à la raison, que les terres ont esté défrichées, \& que les vaisseaux ont fait voile de ce coste 
là, toutes choses y abondent maintenant, \& rien n'y manque, soit pour la necessité, soit pour la délicatesse de la vie. (1667, tome II : 420)

Les Îles que le voyageur tient pour chaotiques sont en effet déjà colonisées. Il ne s'agit pas forcément de montrer que les Autochtones seraient la cause du désordre répugnant qui caractérise le paysage insulaire. Certes, les Îles avaient été laissées aux mains des «Barbares » qui n'avaient pas pris soin de rendre la terre productive. Mais le terme de «barbare » reste flou dans ce contexte et semble renvoyer autant aux précédents habitants européens (conquistadors, flibustiers, engagés et gouverneurs cruels) qu'aux Amérindiens. Encore une fois, la cible de Du Tertre est la négligence des Espagnols qui, préoccupés uniquement par la conquête des continents, ont plongé les Îles dans une instabilité politique dont ont pu profiter notamment les flibustiers. L'autre cible visée par le passage est la politique des autorités françaises, lesquelles n'ont pas suffisamment soutenu la colonie. Et pourtant, malgré les obstacles et contre toute attente, celle-ci a su faire fructifier ces terres « incultes » et préparer la récolte des âmes aussi bien que celle des richesses.

Dans ces passages liminaires, le missionnaire insiste sur les difficultés et les efforts immenses déployés pour surmonter ces obstacles afin de faire de la colonie entière le héros de l'aventure. C'est la représentation imaginaire d'une stratégie réelle que Du Tertre résume ainsi dans une autre partie du livre: «L'isle se peuploit se découvroit, s'embellissoit \& devenoit meilleure de iour en iour.» (1654, tome II : 58) Après des années de guerres, de famines et de maladies, la tyrannie n'existe plus et la paix est conclue avec les Amérindiens.

On en arrive donc à constater avec Geoffroy Atkinson qu'«à la base de l'esprit exotique, d'une façon plus ou moins confuse, se trouve constamment l'idée de progrès, l'idée d'améliorer la condition sociale, politique ou intellectuelle des hommes ${ }^{13}$ ». Atkinson se réfère plus spécifiquement à un exotisme qui emprunte le regard de l'autre pour critiquer sa société dans le but de l'améliorer. Il semble qu'un esprit de progrès analogue influence aussi la représentation de la société française coloniale installée dans les pays lointains - sauf que la situation est quelque peu inversée. Dans le contexte de la relation dutertrienne, le monde extérieur ne sert pas seul de modèle pour améliorer la société européenne. C'est plutôt le contraire. Par sa puissance sublime, le roi de France «porte ses rayons dans ces Pays éloignez » et leur donne une «disposition » qui les rend florissants et fait «naître l'abondance et toutes sortes de biens »; ses sujets, de leur côté, contribuent par les «peines incroyables » qu'ils 
subissent et par leurs « travaux infinis » aux « soins extraordinaires qu'il prend de les conserver », pour créer un nouvel âge d'or ${ }^{14}$.

L'objet de l'étonnement et de l'admiration n'est plus alors la nature ou la société lointaines, mais le développement de la colonie : ce n'est en effet qu'avec l'arrivée des Français qui se mettent au travail et réorganisent l'espace naturel que les Îles prennent leurs traits paradisiaques et deviennent aussi harmonieuses qu'elles étaient potentiellement riches. Tout au long du texte, la portée du travail est relevée comme un éloge indirect de la politique coloniale qui achève le travail de Dieu et répand la grandeur de la France. L'idée que la France embellit les terres lointaines n'est certainement pas unique, mais Du Tertre l'intègre de façon systématique à plusieurs niveaux de sa relation et la place toujours dans un cadre édénique ${ }^{15}$. Les motifs propagandistes sont savamment enveloppés dans un pli du discours paradisiaque qui avait commencé à s'articuler au XVI ${ }^{\mathrm{e}}$ siècle, surtout chez les protestants, et incluent le travail dans la vie édénique ${ }^{16}$. Conçu sous cette forme, l'espace antillais se remplit ainsi d'autres merveilles que la licorne et l'homme à tête de chien: les merveilles du labour de l'établissement. En d'autres mots, il arrache l'exotisme américain à son origine antisociale pour y introduire l'idéal du progrès et proposer ainsi une nouvelle variante de l'Éden : le paradis colonial.

Productivité, travail et beauté sont inséparables dans cette conception de l'étranger, suggérant que la notion de production n'a pas encore pris un sens entièrement utilitaire, mais renvoie autant à la production d'un paysage qu'au profit que l'on pourrait tirer d'une terre ${ }^{17}$. On assiste à la transformation d'un espace naturel en territoire par l'écriture, qui configure celui-ci en un paysage-décor chargé de signification. Ainsi, l'exubérance naturelle et la douceur du climat sont remplacées par la vision d'un autre espace idéalisé, modelé par la main de l'homme. Autrement dit, l'idée d'un paradis colonial repose sur une certaine esthétique; à son fondement se trouve le beau et elle requiert une forme.

L'importance de la mise en ordre ressort également dans les gravures de Leclerc qui illustrent le travail de production aux Îles. Ces illustrations

14 Friedrich Wolfzettel affirme que le voyageur français dénote « un sentiment de supériorité [...] sous l'impact du règne du Roi Soleil ». Le Discours du voyageur, p. 125.

15 Voir à ce propos François de Dainville, qui cite entre autres N. Perrot: « La terre est plus belle depuis que les Français sont venus. » La Géographie des humanistes, p. 477-478.

16 Jean Delumeau cite à ce propos le Treatise of Paradise de John Salkeld qui, lui, reprend Augustin : «Dieu dans sa bonté plaça Adam au paradis, ut operaretur et custodiret illum, pour qu'il puisse travailler et le garder. » Ce travail serait nécessaire au bonheur : «il n'entraînait ni fatigue ni peine mais constituait plutôt un plaisir, $[. .$.$] une récréation et une$ jouissance de la volonté et de l'esprit ». Une histoire du paradis, p. 252-53.

17 Voir Bernard Tocanne, L'Idée de nature en France dans la seconde moitié du XVII e siècle, p. 325 . 


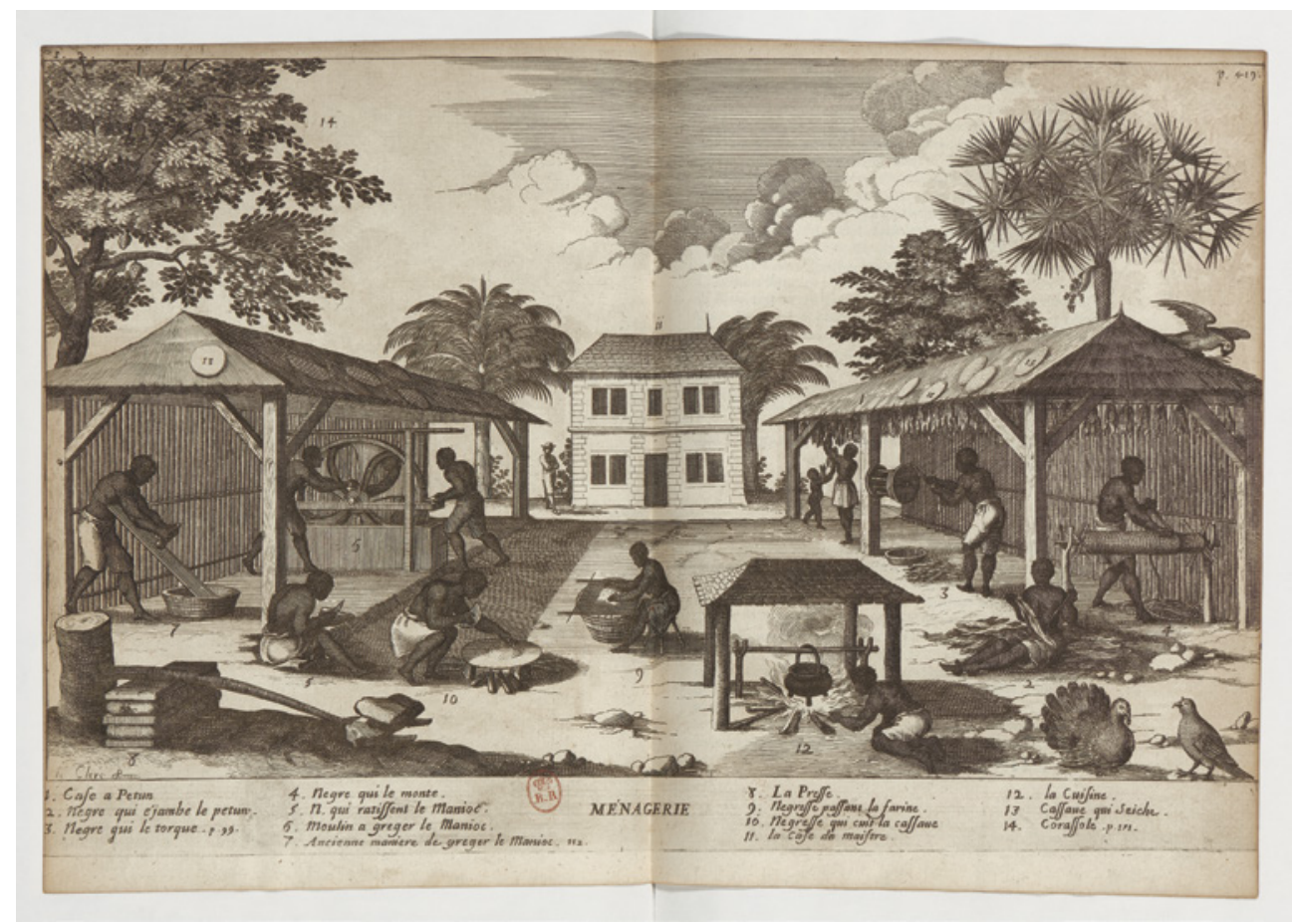

illustration 4 Ménagerie, Sébastien Leclerc

SOURCE GALLICA.BNRF.FR / BIBLIOTHÈQUE NATIONALE DE FRANCE, DOMAINE PUBLIC

complémentent le texte en suivant les normes de l'époque et privilégient «l'esprit de classification au détriment de "l'image choc" d'animaux exotiques rocambolesques », comme le note Sylvie Requemora-Gros ${ }^{18}$. Leur objectif n'est pas de représenter l'étrangeté par le biais d'un spectaculaire qui frapperait l'imaginaire du lecteur, mais plutôt d'offrir l'image d'une vie quotidienne rythmée par la douceur et le calme. Leclerc saisit les modes de productivité de la jeune colonie dans un paysage classique, au fil de trois planches assez connues: la ménagerie, la sucrerie et l'indigoterie, qui représentent la série des étapes de la production du manioc, du tabac, du sucre et de l'indigo. Le travail suit l'ordre qui fait tourner la plantation. Des esclaves, hommes et femmes de tous âges, effectuent le travail ; même si des instruments et des machines sont utilisés, cette organisation mixte se distingue de la mécanisation proto-industrielle que l'on retrouve chez Labat en 1722. Toutela ligne de production, depuisla matière brute jusqu'au produit fini, reflète un mode de vie aux Îles qui serait en harmonie avec la nature.

18 Sylvie Requemora-Gros, Voguer vers la modernité, p. 390. 


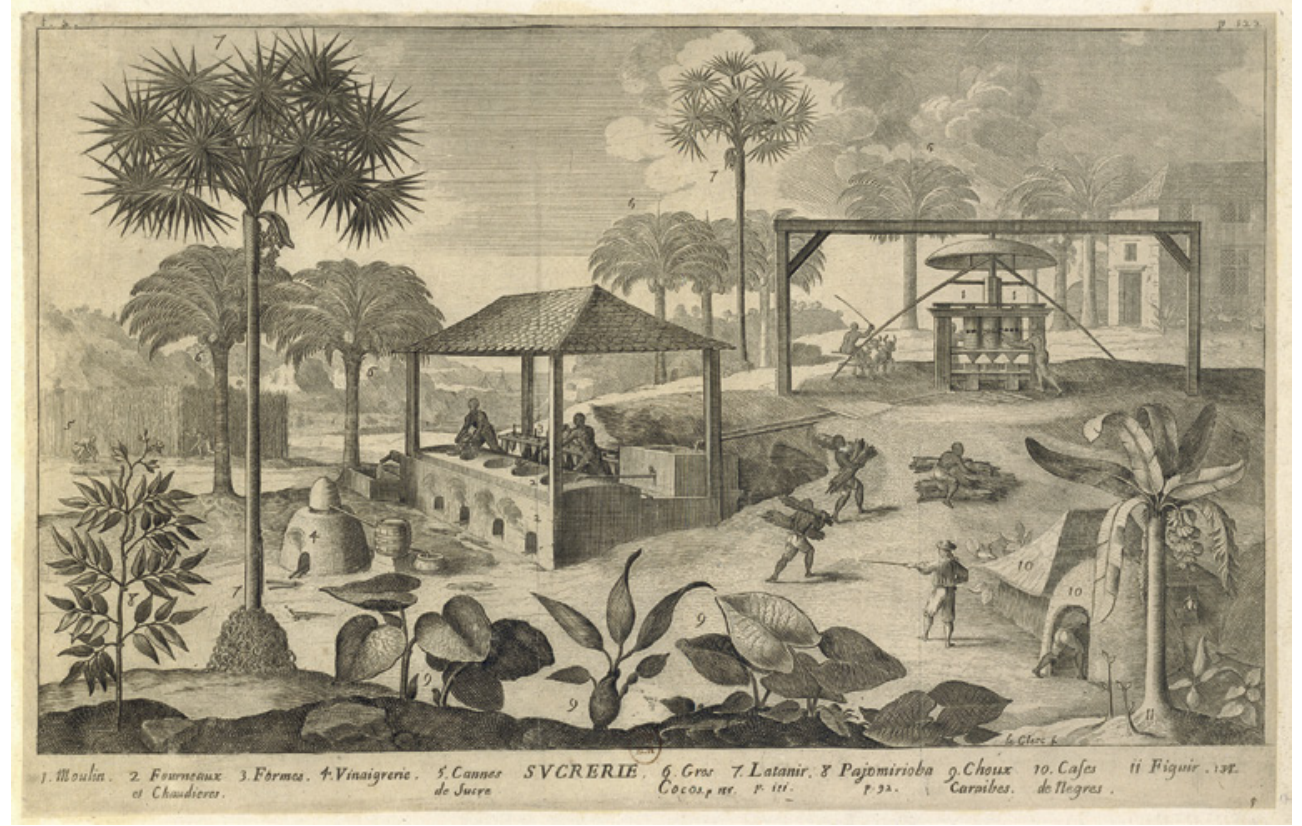

ILlUSTRATION 5 Sucrerie, Sébastien Leclerc

SOURCE GALLICA.BNRF.FR / BIBLIOTHÈQUE NATIONALE DE FRANCE, DOMAINE PUBLIC

La canne à sucre y est représentée, ainsi que le manioc et le pétun, comme ils le seraient sur une planche de botanique. De plus, pour économiser l'illustration, Leclerc insère au premier plan d'autres plantes et arbres exotiques identifiés et nommés. Sur le toit de la ménagerie, on observe un perroquet, dont la présence relève de l'imagerie paradisiaque. Dans chacune des illustrations apparaît un contremaître blanc, habillé à l'européenne et tenant une canne, à la fois menace et rappel de sa supériorité, tandis que les esclaves ne portent que des toiles autour des hanches et travaillent dans le calme, sans signes d'accablement ou de sévices. Toute la production est inscrite dans un paysage qui, à son tour, reflète l'ordre de la colonie et dont la nature inclut l'activité humaine. C'est l'harmonie communautaire du travail compris comme partie intégrante de l'espace naturel qui est au centre de l'attention - et non les machines, comme ce sera le cas dans les représentations de l'industrie sucrière propres au XVIII ${ }^{\mathrm{e}}$ siècle $^{19}$.

19 Déjà, les planches illustrant la production du sucre dans la relation de Labat publiée en 1722 mettent en valeur les machines plus que les hommes qui y travaillent ou le cadre à l'intérieur duquel sont utilisées ces machines. 
Dans cet ensemble communautaire, les cases des esclaves ont aussi leur place à côté de l'habitation du colon, comme pour rappeler à la fois la beauté et la simplicité sauvages ramenées à l'ordre qui s'incarne dans les cases, et le triomphe de la systématisation de l'espace à la française. Le visuel capte la position ambigüe et profondément problématique de Du Tertre face à l'esclavage. Certes, Du Tertre ne cite pas directement la lettre sur les esclaves de Sénèque, mais l'omniprésence des références au philosophe suffit pour afficher l'ambivalence de son propos. Pour lui, l'esclavage pose problème du point de vue éthique et théologique tout d'abord, mais aussi dans la construction de sa vision du paradis colonial.

L'an 1641 marque le début de la traite des Noirs entre l'Afrique et la Martinique, mais le système esclavagiste est encore peu développé dans les îles françaises pendant le séjour de Du Tertre ${ }^{20}$. Il y avait à Saint-Christophe, vers la fin des années 1630, environ 500 esclaves africains achetés du Brésil ou des marchands anglais ou néerlandais, écrit-il. (1667, tome I : 61) Au début de son séjour, la paroisse où il travaille ne possède qu'un esclave, appelé Dominique. Mentionné trois fois, ce personnage n'apparaît qu'en silhouette dans l'Histoire générale des Antilles et, quand Du Tertre décrit les esclaves africains, c'est en termes souvent paradoxaux. On a dit que Louis XIII avait en 1642 permis l'esclavage sur le territoire français, mais à contrecœur, puisque la France, c'est la terre de la liberté. Il l'aurait finalement approuvé sous prétexte que celui-là facilite la conversion des Africains et peut donc « sauver» leur âme. Or, comme le suggère Christopher L. Miller en s'appuyant sur Jean-Baptiste Labat, il s'agit d'une manière de contourner la véritable motivation commerciale de la traite d'esclaves ${ }^{21}$. L'édit n'a jamais été retrouvé, souligne Miller, et il semble bien que l'on ait confondu l'édit permettant la colonisation des Îles avec la légalisation de l'esclavage ${ }^{22}$. L'argument évangélique pro-esclavagiste circulait pourtant à l'époque, mais il est loin d'être facilement accepté par les missionnaires. Il aurait fallu attendre la fin du XVII ${ }^{\mathrm{e}}$ siècle, l'avènement de l'agriculture sucrière et

20 Le premier recensement des esclaves et des habitants des îles date de 166o. Voir Philip Boucher, France and the American Tropics, p. 238-239. Selon Du Tertre, il n'y avait que huit ou neuf esclaves à Saint-Pierre autour de 1642. (1667, tome II : 27).

21 Christopher L. Miller, The French Atlantic Triangle: Literature and Culture of the Slave Trade, Durham NC, Duke University Press, 2008, p. 18-19.

22 Voir Sue Peabody, There are no Slaves in France: The Political Culture of Race and Slavery in the Ancien Régime, Oxford, Oxford University Press, 1996, p. 176. Voir aussi Labat, Nouveau voyage, vol. IV, p. 114. 
le développement considérable de la traite qu'il entraîne, pour que cet argument s'impose ${ }^{23}$.

Le regard de Du Tertre sur les esclaves reste dominé par la vision universaliste chrétienne selon laquelle tous les hommes sont égaux, partageant un destin commun, sous le regard de Dieu ${ }^{24}$. Du Tertre, qui suit Las Casas, soutient que les Autochtones ne devraient pas être soumis à l'esclavage; (1667, Tome II : 454) de même, il n'est pas entièrement convaincu que la solution au besoin de main-d'œuvre se trouve dans l'asservissement des Africains. En fait, l'Histoire générale des Antilles oscille entre deux postures contradictoires face à l'esclavage. D'un côté, l'expression, toute en comparaisons et en métaphores, d'un sentiment d'horreur envers un système qui exploite les créatures de Dieu, et la pitié qui en résulte pour celles-ci. En même temps, il défend dans l'édition de 1667 les habitants contre les accusations qu'on leur fait en France en disant qu'ils soumettent des hommes à l'esclavage dans « un Païs où ils vivent selon les loix de France, qui abhorre la servitude sur toutes les Nations du monde. » (1667, tome II : 483) Les richesses des Îles, dont jouissent aussi les métropolitains, viennent de l'esclavage, constate-il et dénonce par-là indirectement l'hypocrisie de la position européenne comme un Voltaire. Cela dit, Du Tertre témoigne aussi de sa conviction que l'esclavage pourrait mener au salut des Africains, pourvu qu'ils se convertissent. Mais cette idée est en elle-même pleine de contradictions: si les esclaves sont convertis, il devient impossible de justifier l'assujettissement brutal auquel on les soumet ${ }^{25}$. C'est là un argument qui revient tout au long du siècle suivant dans les débats autour de l'esclavage, comme l'a démontré Michèle Duchet ${ }^{26}$. Sur les îles anglaises, ce sont les autorités civiles qui résolvent la situation en refusant aux esclaves le baptême. Les missionnaires français, pour leur part, ne sortent jamais du dilemme et restent piégés entre raison économique et impératif théologique. Mais on voit déjà chez Du Tertre que la raison économique gagne du terrain. De peur que les esclaves ne se révoltent, il conseille par exemple d'éviter de leur donner trop d'instruction; il faut qu'ils restent à leur place. La conversion

23 On le voit clairement dans les lettres du jésuite Jean Mongin. Arrivé en Martinique en 1682, il fut chargé de convertir les esclaves. Lettres du R.P. Jean Mongin. L'évangélisation des esclaves au XVII e siècle, Bulletin de la Société d'Histoire de la Guadeloupe, Marcel Chatillon (dir.), Point-à-Pitre, 1984, p. 61-62.

24 Voir l'excellente analyse de Joan-Pau Rubiés « Were Early Modern Europeans Racist? » Ideas of "Race" in the History of the Humanities, Amos Morris-Riech et Dirk Rupnov (dir.), New York, Palgrave Macmillan, 2017.

25 Voir Sue Peabody, "A Nation Born to Slavery": Missionaries and Racial Discourse in Seventeenth-Century French Antilles », Journal of Social History, vol. 15 (1), 2004, p. 113-126. 
ne doit jamais aller trop loin, au risque de nuire à la production. L'expression de la compassion à l'égard du sort des esclaves n'est donc pas rare sous la plume des missionnaires, sans pour autant que le sentiment ne débouche sur un discours contre l'esclavage en tant que tel ${ }^{27}$.

Pour escamoter le dilemme théologique, Du Tertre construit une version plus «humaine» de l'esclavage, fournie là encore par l'imaginaire paradisiaque. Dans certains passages, le relateur contemple avec attendrissement les petits esclaves dont il décrit les jeux:

C'est un plaisir nompareil que de voir trois ou quatres petits Négres se joüer ensemble pendant que leurs meres travaillent, car ils se barboüillent, se renversent, \& sont tantost dessus, tantost dessous; sans pourtant se faire aucun mal, si bien qu'ils ne crient point, \& ne détournent point leurs meres de leurs bensonges, si ce n'est pour leur donner à téter. (1667, tome II : 509)

On est là loin des horreurs réelles du travail forcé. La description rappelle une toile bucolique où le missionnaire contemple le jeu de petits enfants noirs au lieu de moutons. Tout comme les Autochtones, les esclaves font partie intégrante de la nature selon cette vision, mais non pas de la nature sauvage. Bien au contraire, les Africains s'inscrivent dans la nature domestiquée par l'établissement et jouissent ainsi, selon Du Tertre, d'un bonheur qui se refléterait dans l'espace où ils vivent: « Leurs Cases ont du rapport à celles où se retiroient les habitans du siecle d'or, dont Seneque nous décrit le bonheur. » (1667, tome II : 517) Le discours d'Hippolyte tiré du Phèdre de Sénèque, souvent considéré comme la version mondaine du mythe de l'Éden terrestre, se voit ainsi plaqué sur la réalité misérable de la condition servile, afin de célébrer le profit que tireraient du paradis colonial même ceux qui sont forcés de participer par leur corps et leur travail à l'ordre qui y règne - un ordre dont la description suggère qu'il produit plus de bonheur pour ces esclaves aux Antilles que ne leur en donnerait l'état de liberté en Afrique.

Dans un autre passage, c'est un cadre biblique qui sert à édulcorer la souffrance des esclaves :

Les femmes Négres sont naturellement fort fecondes, si bien qu'il semble que Dieu renouvelle en leur personne la merveille des femmes Iuives esclaves en Egypte: car plus elles ont de mal, \& plus elles ont d'enfans; \&

27 Voir Lynn Festa, Sentimental Figures of Empire in Eighteenth-Century Britain and France, Baltimore, Johns Hopkins University Press, 2006. 
le seul amour qu'elles leur portent les empesche d'en avoir davantage [...] (1667, tome II : 505)

Le relateur reconnaît donc l'état de misère dans lequel vivent ces femmes et le rapporte à une prévoyance divine en le resituant dans le cadre de l'Écriture ; il en transforme ainsi le résultat - la fécondité causée par le malheur - en une preuve supplémentaire de l'existence d'un paradis colonial. Le même cadre de référence revient lorsqu'il cherche à illustrer à quel point le supplice des esclaves est exceptionnel :

Si le travail, auquel Dieu engagea le premier homme, est un chastiment de sa rebellion; \& si la justice vangeresse y a tellement obligé les mal-heureux enfans de ce Pere coupable, que Iob asseure qu'il ne leur est pas moins naturel, que le vol d'oyseau: on peut dire que les Négres souffrent la plus rigoureuse peine de cette revolte. (1667, tome II : 523)

Tout en laissant entrevoir l'atrocité du travail forcé, Du Tertre en montre le principe comme une conséquence naturelle de la chute et transforme ainsi la logique économique de l'esclavage en argument théologique ${ }^{28}$. À cela s'ajoute aussi un argument d'ordre protoanthropologique : si le désir de liberté est commun à tous les hommes, il le serait moins chez les esclaves, qui sont prédestinés à servir. Son raisonnement dilue la question de l'assignation à une ethnie particulière de cette caractéristique en faisant remonter l'argument à Platon, chez qui l'esclavage n'est pas déterminé par l'origine des peuples : «Un Poëte avoit bien raison de dire chez Platon, que Dieu oste la moitié de l'esprit aux esclaves [...] de peur que connoissant le malheur de leur condition, ils ne s'en affligent avec excez, \& ne deviennent incapables de rendre service.» (1667, tome II : 526) Au lieu d'adresser directement l'esclavage qui se déroule devant ses yeux et sous son autorité, il le déplace du contexte transatlantique et l'inscrit dans l'héritage antique.

Du Tertre ajoute pourtant une dimension à la servitude théoriquement pré-ordonnée des esclaves en suggérant que la véritable source de la souffrance de ceux-ci viendrait du fait qu'ils ont été exclus de la motivation fondamentale du projet colonial, puisqu'ils ne peuvent tirer aucun profit de leurs peines. Leur travail est infructueux et ils sont tout à fait conscients que « toutes leurs sueurs vont au profit de leurs Maistres, \& que quand ils leurs amasseroient des montagnes d'or, il ne leur en reviendra jamais rien, \& que quand ils

28 Voir Réal Ouellet (dir.), La Colonisation des Antilles, p. $5^{28}$. 
vivroient des siecles entiers, \& qu'ils travailleroient davantage qu'ils ne font, ils ne retireroient pas un sol de profit de toutes leurs peines ». (1667, tome II : 525)

L'esclavage constitue donc un problème pour Du Tertre non seulement parce que certains esclaves sont chrétiens, mais parce que leur condition servile les empêche de prendre part à sa vision progressiste du paradis colonial. Par conséquent, les scènes touchantes montrant des esclaves heureux, même momentanément, interviennent pour créer une version alternative de la vie coloniale dans laquelle les esclaves contribueraient par leur travail physique et par leur fécondité à la prospérité générale, et seraient récompensés par l'entremise d'une habitation qui pourvoirait à tous leurs besoins. L'harmonie du paradis colonial repose alors toujours sur une hiérarchie stricte, mais présentée comme naturelle : une tâche y est assignée à chacun, et elle n'est pas le résultat d'un abus de pouvoir. Le travail dans ce cadre cesse d'être cause de souffrance, ce qui permet au missionnaire d'éviter le problème posé par la brutalité et par l'injustice de l'asservissement. Ainsi parvient-il à créer la fiction d'une servitude qui répondrait aux besoins d'esclaves prédestinés par leur nature à ce sort, niant la souffrance causée d'un côté par la sujétion forcée, et de l'autre par les mauvais traitements subis. Les passages qui saisissent le paradis colonial relèvent de l'éloge d'une vie simple et champêtre où le travail devient source de prospérité économique pour la nation et de bonheur pour l'individu, dans lequel l'imaginaire sauvage et primitif se superpose au rêve archaïque ${ }^{29}$.

Du Tertre fait entrer le travail dans le processus de domestication qui inclut l'étrangeté et qui permet l'émergence d'une nouvelle forme de paradis. Son exotisme conjugue ainsi ce que Bernard Tocanne voit comme une dualité typique de la pensée du XVII ${ }^{\mathrm{e}}$ siècle, dont celle-ci resterait elle-même inconsciente, et qui consisterait en «une démarche qui part des faits pour analyser les conditions réelles dans lesquelles vivent et survivent les sociétés, et une démarche critique qui nourrit des rêveries sur la nature primitive de l'homme, sur la construction d'une cité idéale conforme à une nature perdue et enfin retrouvée ${ }^{30}$ ». La description des conditions réelles de la vie aux Îles trouve dans les parties paratextuelles internes de l'Histoire des Antilles un espace idéal où peuvent se déployer les scènes d'une vie coloniale utopique sur les ruines du primitivisme sauvage.

29 Voir Bernard Tocanne, L'Idée de nature en France dans la seconde moitié du XVII ${ }^{e}$ siècle, p. 199. À côté de la science naissante, il existe des rêves utopistes et primitivistes chez Fleury, Fénélon et bien d'autres.

Ibid., p. 206. 